\title{
Boltzmann Machine Algorithm based Learning of OLSR Protocol: An Energy Efficient Approach
}

\author{
Ashish Kots \\ Department of Computer \\ Science \\ Amity University \\ Noida, India
}

\author{
Vijeta Sharma \\ Department of Computer \\ Science \\ Banasthali University \\ Rajasthan, India
}

\author{
Manoj Kumar \\ Department of Computer \\ Science \\ Amity University \\ Noida, India
}

\begin{abstract}
In this paper, a proposal for OLSR protocol based upon Boltzmann learning algorithm is made. The main focus is to tune OLSR by using Boltzmann learning algorithm. The proposed work deals with the standardized OLSR routing protocol, to make it more reliable, more energy efficient and more adaptable to the rapidly changing network topology and infrastructure. This article therefore provides a simple mechanism for dynamic adoption of mobile nodes based on Boltzmann learning algorithm with OLSR. Then the paper provides a mathematical proof how Boltzmann Learning can be used in MANETs using OLSR.
\end{abstract}

\section{General Terms}

Computer Network, Routing

\section{Keywords}

MANET, Boltzmann, OLSR, routing

\section{INTRODUCTION}

In today's fast moving world, there is a need of the medium that keep channels of communication alive. Therefore set of rules required that can provide us unlimited mobility. Mainly Mobile Ad-hoc Network (MANET) is used to provide unlimited mobility. MANETs [1] are combination of various mobile devices or networks connected wirelessly in an ad-hoc manner. MANETs provides better flexibility in terms of usage but due to large number of mobile nodes there are continuously rapid changes in the network topology too. This degrades the performance of the whole network and manual changes in the network required to provide better performance. But the matter of concern here is how to identify the best protocol for such type of networks that provides best services to the communication channel in any situation and environment. The self-configuring nature of MANETs is supported by these three basic protocol standards:-

- $\quad$ AODV (Ad-Hoc on Demand Distance Vector) RFC 3561

- $\quad$ DSR (Dynamic Source Routing) RFC 3684

- $\quad$ OLSR (Open Link State Routing) RFC 3626

Here Optimized version of Link State Routing protocol is taken into consideration as it carries the best features of several other routing protocols. Its performance \& characteristics has been studied expensively [2-4].

\section{OLSR PROTOCOL}

In this section some basic features of OLSR protocol are discussed. The Optimized Link State Routing protocol is a table-driven proactive protocol. It is based on the Link State Routing and here nodes in the network frequently share their link state \& topology information with all the other nodes present in the network by using the concept of multipoint relays (MPR). By using the MPR concept, optimized flooding and continuous updating of the network infrastructure is done. Selective flooding increases the network performance and as a node changes its states its information is updated in the network.



Fig 1: A MPR (Black Nodes) based multi hop network for flooding a packet

\section{BOLTZMANN MACHINE}

Discrete Hopfield network with simulated annealing process is known as Boltzmann machine [5]. It is a massive computational organization that operates in a parallel computation environment and provides effective solution to the networks that can even involves large number of weak constraints. States of all the units are represented by a vector of state and these states are responsible for the configuration of the network. These states of the units are represented by binary value with probabilistic state transitions. The Boltzmann machine is composed of interconnected elements called units and they have bidirectional links for the communication. A unit can adopts one of the states either ON or OFF and to acquire these states neighbouring units are used with a probabilistic function and weights on the links [6, 7]. A 
unit currently accepts or rejects some information about the domain is decided by its state ON or OFF.

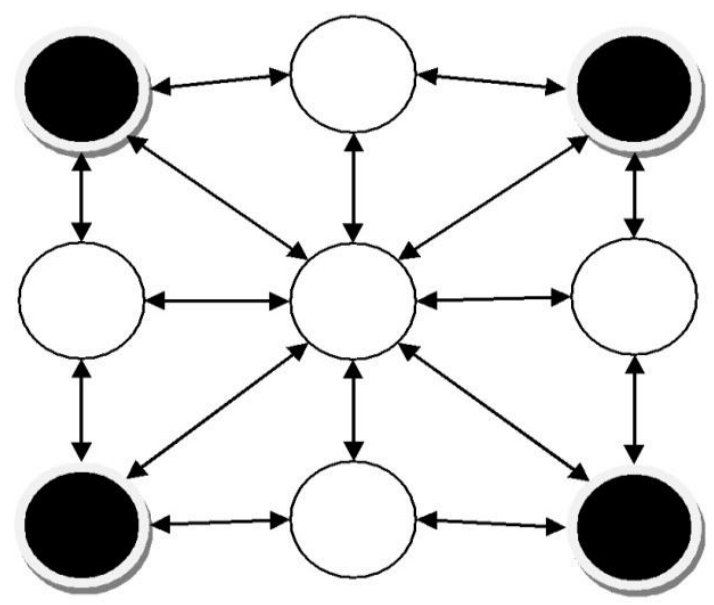

Fig 2: Architecture of Boltzmann Machine with ON (Black Nodes) and OFF (White Nodes) States

\section{PROPOSED WORK}

The processing ability of massive networks mainly depends on the communication bandwidth between the various networking elements and the interconnection between them. These processing elements are responsible for the packet forwarding from on node to another in the network. In this article a proposal for a learning mechanism that is based on statistical mechanics and can be used as a learning rule for the modification of connection strengths in OLSR and it also provides an energy efficient routing for the MANETs. The proposed work uses strengths of the interconnection between various communicating elements and provides effective communication using the physical interconnection [8].This article presents a Boltzmann machine learning based OLSR for MANETs that uses constraints to satisfy the network needs and capable of learning in the boundary of defined constraints. The network here strengthened its interconnection to generate an internal model using probability distribution. At present, an interesting mathematical result has been shown in favour of the proposed article.

\subsection{BOLTZMANN BASED OLSR}

In Boltzmann based OLSR all the nodes present in the network are composed of primitive computing elements called units and connection strength between two nodes is represented as the weights on their links. All the interconnected nodes have bidirectional links means communication can be done in either ways. A node can have one of the two states either ON or OFF and these states are based on the states of the neighbouring units and the connection strengths between them. Nodes use probabilistic function to change its states from $\mathrm{ON}$ to OFF or from OFF to ON. In a network domain a node is ready to accept or reject some information is based on the unit being ON or OFF. The weight on an interconnection represents a pair wise constraint between two units or nodes. A positive weight indicates that connection is currently active and both the interconnecting unit tends to support each other. A negative weight indicates that connection between the two nodes is down and currently they are not able to communicate.

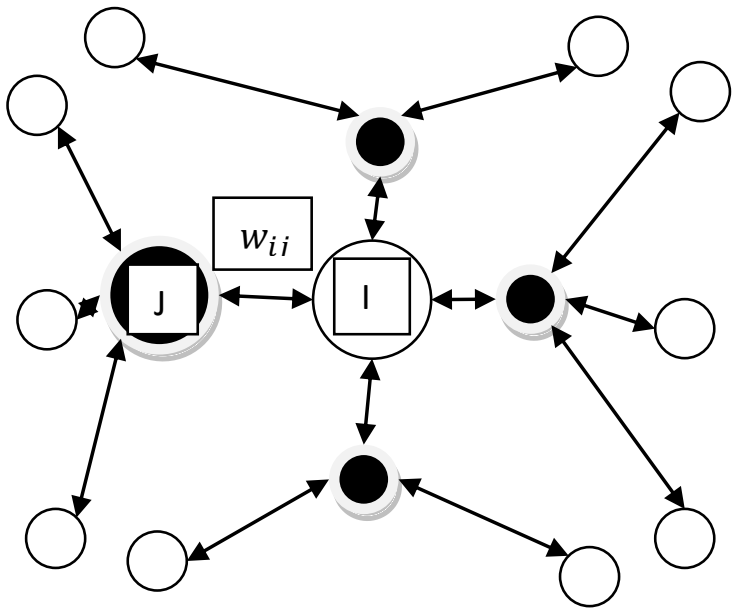

Fig 3: A representation of Boltzmann based OLSR

The resulting network resembles to the Hopfield Network [9], and as in the Hopfield system, the Boltzmann based OLSR network can be assigned a global "energy" state. In this approach, first configuration of the MPRs and all other nodes has to be done in such a way that they are able to minimize the global energy. In the initial phases if some of the nodes or MPRs are forced to clamp their states to accept or represent a particular but after some alteration system will be able to find a configuration with a minimum energy for that particular input. In this way all the node can be tuned in the OLSR with a minimum energy extent that will guarantee to an energy efficient OLSR for MANETs. The global energy state for the Boltzmann based OLSR is defined as

$$
E=-\sum_{i<j} w_{i j} s_{i} s_{j}+\sum_{i} \theta_{i} s_{i}
$$

Here $w_{i j}$ is the strength of interconnection between two nodes $\mathrm{i}$ and $\mathrm{j}$ in the network and $s_{i}$ is 1 if is ON otherwise zero and $\theta_{i}$ is the minimum threshold for every node in the network. If all the nodes or a unit in the network works in an asynchronous manner and their transmission time is also minimal, then the whole network always holds a local energy minimum [9]. As the connection strength acts in the same fashion in the both direction the energy gap for the $\mathrm{k}^{\text {th }}$ unit is given by

$$
\Delta E_{k}=\sum_{i} w_{k i} s_{i}-\theta_{i}
$$

This shows that the condition to minimize the energy of a node or unit contributed to the global energy state and to adopt the ON state, its total input have to exceeds its threshold value. As a MPR node in the network goes down to its threshold value it sets its states from ON to OFF state and a new MPR node is selected from the MPR set. By this way the OLSR always maintains an energy efficient state and produces efficient results in comparison to the previous version on OLSR. This threshold condition can be applied to all the present nodes in the network. To tune OLSR a bias $-\theta_{i}$ is provided, known as TRUE UNIT and it is used to simplify the computations as it is treated as the links between two nodes. If this bias treated as a part of every network Eq.1 $\& 2$ becomes:- 


$$
E=-\sum_{i<j} w_{i j} s_{i} s_{j}
$$

$$
\Delta E_{k}=\sum_{i} w_{k i} S_{i}
$$

Let the gap in energy between two states of a node (ON and $\mathrm{OFF})$ is $\Delta E_{k}$ and $\mathrm{T}$ is temperature parameter of the node, then the probability $\mathrm{P}_{\mathrm{k}}$ regardless of the previous state set $\mathrm{S}_{\mathrm{k}}$ is given as

$$
p_{k}=\frac{1}{\left(1+e^{-\Delta E_{k} / T}\right)}
$$

A network in which all the nodes or units follow the above decision rule to set it sates ON or OFF will eventually reach the minimal global energy state known as thermal equilibrium condition for all the nodes and then Boltzmann distribution is followed by all the global states of the network and their probability is given as:

$$
\frac{P_{a}}{P_{b}}=e^{-\left(E_{a}-E_{b}\right) / T}
$$

Here $\mathrm{P}_{\mathrm{a}}$ and $\mathrm{E}_{\mathrm{a}}$ represent the probability respectively of the $\mathrm{a}^{\text {th }}$ global state and energy state.

\subsection{LEARNING MECHANISM OF BOLTZMANN BASED OLSR}

The best part of Boltzmann based OLSR is that it moves in an independent manner is such a way that it develops an internal learning mechanism that have the ability to modify the connection strength between the nodes in the network and modify the network structure of its environment. By using the above equations (1), (5), (6) an interesting relationship between the global energy states and the connection strengths between the nodes of the network have been found:-

$$
\frac{\partial \ln P_{a}}{\partial w_{i j}}=\frac{1}{T}\left[s_{i}^{a} s_{j}^{a}-P_{i_{j}}\right]
$$

In the above equation $s_{i}^{a}$ represents the state of the $\mathrm{i}^{\text {th }}$ node in the network and having $a^{\text {th }}$ global state and $P_{i j}$ is the probability of two nodes at $\mathrm{ON}$ state in at the equilibrium state of the network. Using the above relationship nodes of OLSR can easily modify their connection strengths according to the network infrastructure. In the initial state of learning probabilities of the global state of the network have to be provided explicitly. Later on network becomes adoptable and manage the connection strength \& energy state itself.

\subsection{STRUCTURE OF BOLTZMANN BASED OLSR}

The nodes of a Boltzmann Based OLSR are grouped in this way:-

\subsubsection{Visible nodes set}

A nonempty set of nodes that are currently active in the OLSR based network.

\subsubsection{Hidden nodes set}

A possible empty set of nodes that are currently not a part of the OLSR based network.
The visible nodes acts as the basic interface between the OLSR based network and the environment and are clamped from $\mathrm{ON}$ to $\mathrm{OFF}$ or OFF to $\mathrm{ON}$ during the training of the OLSR. After reaching the thermal equilibrium state only MPR node from the set of MPRs are often clamped. As an entry arrives in the Hidden node set all the MPRs update information in their repository.

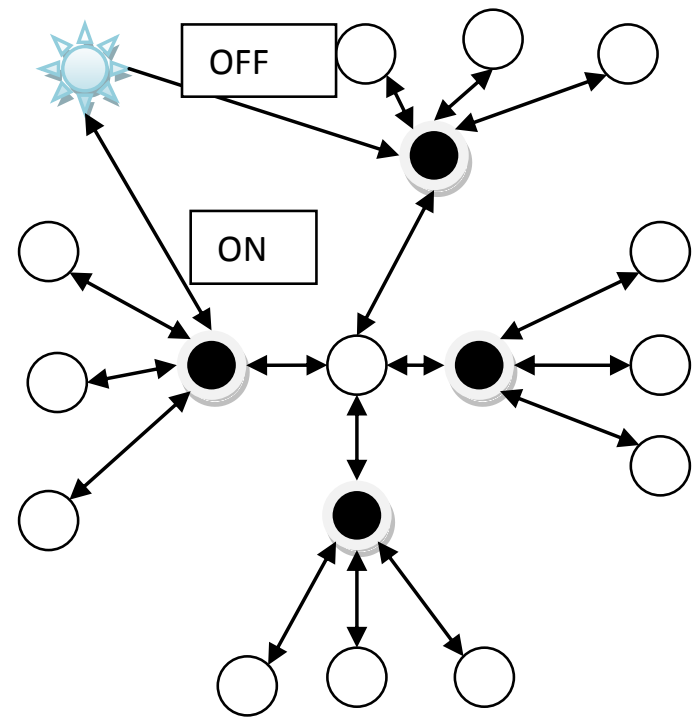

Fig 4: A scenario of visible nodes and hidden nodes in Boltzmann based OLSR

Whenever an entry is made in the hidden unit set of the Boltzmann based OLSR its information is updated in the one of the MPR repository and its current state is set to ON for this MPR and OFF for the rest of MPRs present in the network. This mechanism can help in the authentication of the hidden nodes and also customize the structure of the OLSR based network.

A mathematical representation between this internal network and the environment is given by:-

$$
G=\sum_{\alpha} P\left(V_{\alpha}\right) \ln \frac{P\left(V_{\alpha}\right)}{P^{\prime}\left(V_{\alpha}\right)}
$$

Here $G$ is called the information gain [10] [11] from the network. As probability in the all the equations dependent on the connection strength, so information gain be modified or changed using them. By using the Eq. 1 \& 6 partial derivative of $\mathrm{G}$ is derived:-

$$
\frac{\partial G}{\partial w_{i j}}=-\frac{1}{T\left(P_{i j}-P^{\prime}{ }_{i j}\right)}
$$

In the above equation $\mathrm{P}_{\mathrm{ij}} \& \mathrm{P}_{\mathrm{ij}}$ respectively represents the probabilities of the two nodes in the ON state when network is changing the state of visible nodes and when the network is running in the free mode without the environmental effects. Now using these two probabilistic conditions of the nodes one can easily do updating in their connection strength using the following:-

$$
\Delta W_{i j}=\varepsilon\left(P_{i j}-P^{\prime} i_{j}\right)
$$

The change in the connection strength is totally depends on the connection between two nodes and their connection to all 
the corresponding visible nodes present in the network. As soon as information gain function is finalized and then minimized the Boltzmann based OLSR will be able to perform all the regularities in the network and using them it will start to adopt the network itself.

\section{CONCLUSION}

The Boltzmann based OLSR protocol for MANETs provides a distributed representation in terms of the minimum energy and it also adopts any environment and configures itself by using information gain function of the network and will provide best connection strength for the information sharing. The main drawback of this learning OLSR is that in the initial phase of learning its performance degrades until it reaches the thermal equilibrium state for the whole network.

\section{REFERENCES}

[1] S. Carson, J. Macker, "MANETs (MANET): Routing Protocol Performance Issues and Evaluation Considerations". Network Working Group. RFC 2501. January 1999.

[2] R.S.SISODIA, B.S.MANOJ and C.S.R.MURTHY. "A preferred link-based routing protocol for ad hoc wireless networks". Journal of Communications and networks, 4(1):14-21, March 2002

[3] T. Clausen, P. Jacquet, L. Viennot, "Comparative Study of Routing Protocols for Mobile Ad-hoc Networks," Proceedings of IFIP Med-Hoc-Net 2002, September 2002
[4] T. Clausen, P. Jacquet, A. Laouiti, P. Minet, P. Muhlethaler, A. Qayyum, L.Viennot, "Optimized Link State Routing (OLSR) Protocol," RFC 3626, http://ietf.org/rfc/rfc3626.txt,2003.

[5] David H. Ackley, Geoffrey E. Hinton, Terrence J. Sejnowski, "A Learning Algorithm for Boltzmann Machines”, Cognitive Science 9, 147-1699(1985)

[6] Rich Caruana, "Multitask Learning", Machine Learning, 28(1):41-75, 1997

[7] R.Salakhutdinov,"Learning and evaluating Boltzmann Machines", Technical Report UTML TR 2008-002, Dept. of Computer Science, University of Toronto, June 2008

[8] S.E. Fahlman, G.E. Hinton, T.J. Sejnowski,"Massively parallel architectures for AI: NETL, Thistle, and Boltzmann Machines", Proceedings of the National Conference on Artificial Intelligence AAAA1-83, Washington, DC, 109-113

[9] J.J. Hopfield, "Neural networks and physical systems with emergent collective computational abilities", Proceedings of the National Academy of Sciences USA, 79, 2554-2558, 1982

[10] S. Kullback, “ Information theory and statistics", New York: Wiley, 1959

[11] A. Renyi, "Probability Theory", Amsterdam: NorthHolland, 1962 